\title{
Research on the Application of Artificial Intelligence in Administrative Governance
}

\author{
Yiling Cao \\ School of Law, Humanities and Sociology \\ Wuhan University of Technology \\ Wuhan, China
}

\begin{abstract}
The advent of the artificial intelligence era has changed the system and mode of various fields of society, and the intelligent era has become the new background of modern administrative governance. The new stage of artificial intelligence development will make it an effective means to promote the intelligentization of administrative governance on the basis of changing human production and life style. The integration of artificial intelligence into administrative governance makes the administrative governance system continuously optimized, but the uncertainty of artificial intelligence development still exists. It also brings many realistic shocks and challenges to the administrative governance system and the government's ability to govern. Can the existing administrative governance level of the administrative organs cope with the new risks and uncertainties brought about by artificial intelligence, and how to effectively apply artificial intelligence to administrative governance? The use of regulation to build a new pattern of administrative governance has become an urgent problem to be solved. Based on the intelligentization of administrative governance, this paper expounds and analyzes the application of artificial intelligence in administrative governance, and considers how to regulate the application of artificial intelligence in administrative governance.
\end{abstract}

Keywords—artificial intelligence; administrative governance; administrative functions

\section{INTRODUCTION}

The concept of "artificial intelligence" (referred to as AI) is currently defined by the academic community. According to the relevant research of foreign scholars, it is generally defined as a branch of computer science, which is a collection of theories, methods, techniques and application systems. Technical science, mainly used to research, develop, simulate, extend and extend human intelligence. According to its development degree, artificial intelligence can be divided into three stages: weak artificial intelligence, strong artificial intelligence and super artificial intelligence. At present, the widespread application of intelligent machines in the fields of computer and industrialization makes weak artificial intelligence have been realized, from the current artificial intelligence technology. In terms of development, intelligent machines do not have self-awareness, and there is no strong artificial intelligence and super artificial intelligence. However, with the gradual maturity and extensive application of artificial intelligence technology, "weak artificial intelligence" is continuously being delivered to the "strong artificial intelligence" stage.

The advent of the era of artificial intelligence has made artificial intelligence carriers one of the main bodies of multi-governance, and promoted the continuous development of administrative governance systems. At the same time, the use of intelligent governance has enabled artificial intelligence technology to have the ability to solve governance problems independently. In the era of traditional governance, administrative governance subjects can only formulate limited governance programs due to incomplete data and other reasons, and cannot accurately predict potential risks in the governance process, which makes administrative governance more difficult, and governance faces many uncertain risks. . The development of the Internet and big data has improved the integrity and convenience of information collection of administrative subjects. On the basis of this, through the powerful algorithm function of artificial intelligence technology, the administrative governance subject can generate scientific and reasonable specific governance plans in a relatively short period of time. Difficulties and risks have been reduced compared to traditional governance, and administrative governance has been rapidly improved. However, the application of artificial intelligence in administrative governance is also facing enormous challenges. The shortcomings and defects of artificial intelligence technology will affect the accuracy of administrative governance to a certain extent. Therefore, the administrative subject uses artificial intelligence technology to enrich administrative governance, improve administrative efficiency, and solve the problems arising from the application of artificial intelligence in administrative governance in the dilemma of administrative governance.

\section{PROBLEMS AND REgUlation PATHS OF ARTIFICIAL INTELLIGENCE IN ADMINISTRATIVE GOVERNANCE}

\section{A. Problems in the Application of Artificial Intelligence in Administrative Governance}

First of all, the data security of artificial intelligence itself is not high. Data and algorithm are important components of artificial intelligence technology. Artificial intelligence algorithm is essentially "in the form of mathematical or 
computer code." For artificial intelligence management systems based on intelligent machines, control data controls the algorithm. The more data you have, the more accurate the algorithm is. When the data is not comprehensive and inaccurate, data discrimination will occur. The artificial intelligence data may lack balance and representativeness, which makes the data biased and the data security not high. The omission, deviation or lag of the data may cause the representativeness of the data samples to be missing, resulting in unpredictable results of the calculated algorithm. To some extent, it affects the fairness of the algorithm. In the process of public security intelligent monitoring and risk prevention, administrative agencies may have data bias. Therefore, when using predictive algorithms in specific situations, basic data sources, data information and data should be used to some extent. Use methods to disclose to ensure the accuracy and security of the data. Artificial intelligence data and algorithm operation security issues will affect network security, privacy and security. Article 76 of the "Network Security Law of the People's Republic of China" stipulates: "Network security refers to preventing attacks on the network by taking necessary measures. Intrusion, interference, destruction and illegal use, and accidents, the network is in a stable and reliable state, and the ability to guarantee the integrity, confidentiality and availability of network data." Article 45 of the "Network Security Law of the People's Republic of China" stipulates that "the departments and their staff members who are responsible for the cyber safety supervision and management duties must strictly keep confidential the personal information, privacy and trade secrets that are known in the performance of their duties, and must not disclose, sell or illegally provide them to others." The use of artificial intelligence technology in the process of administrative governance should ensure the accuracy and controllability of data and algorithms, and ensure the security of network systems and the privacy of relatives.

Second, the use of artificial intelligence leads to the lack of technical ability of the governance subject. With the gradual expansion of the role of artificial intelligence in administrative governance, artificial intelligence technology is constantly embedded in administrative governance, and artificial intelligence is widely used in various fields of administrative governance, accelerating the process of administrative governance. However, the application of artificial intelligence technology can bring favorable conditions to administrative governance and improve the quality of public services. At the same time, it may cause a "technical gap" between the administrative department and the society, resulting in the lack of technical ability of the governance subject. In the face of the extensive use of artificial intelligence technology in the commercial market and various fields of society, the administrative organs should improve their technical skills to cope with this phenomenon. Otherwise, there will be a very obvious technical gap. When the administrative organs use artificial intelligence, they need complete information. The support of the data system, that is, the entire government process should realize the complete unity of informationization and dataization, information and data resources, and it is possible to establish an effective government intelligence system. It is not enough to introduce intelligent systems alone. As an emerging technology, artificial intelligence is used in administrative governance, which can improve the efficiency of administrative governance to a certain extent. However, at the root of it, artificial intelligence is only an auxiliary means and means for administrative governance and discretion. The administrative subject cannot be a technical carrier for administrative governance. Take the lead and ignore the technical ability of the governance subject itself.

Then, the use of artificial intelligence accelerates the weakening of administrative governance. The administrative organs in the era of traditional governance are the core of the administrative governance system and the makers of administrative governance rules. The advent of the era of artificial intelligence has made data and algorithms more and more important in administrative governance. The makers of big data and core algorithms have gradually become the dominant players in administrative governance. The administrative agencies in the era of artificial intelligence still have practical administration. Power, but with the artificial intelligence technology deep into the administrative governance system, the administrative agencies rely on the lack of data collection, use and the use of artificial intelligence technology and research and development capabilities, the administrative agencies continue to rely on the advantages of big data and artificial intelligence technology business enterprises Deepening, because the artificial intelligence system may be designed and developed by the market subject, the administrative subject lacks the understanding of the system algorithm and running program of the intelligent machine when using artificial intelligence. The opacity of the intelligent system algorithm may cause a large amount of data information to be under the control of the background, the market participants are aware of the obvious advantages of data. These data are extensive, and the enterprise entities can use these data to implement their own behaviors without being controlled. The application of artificial intelligence in administrative governance will weaken the governance ability of administrative subjects to a certain extent.

At last, intelligent governance creates the dilemma of administrative ethics. The wide application of artificial intelligence poses a challenge to traditional administrative ethics, and at the same time it also triggers a new administrative ethical dilemma. Human administrative ethics follows a basic principle. The exercise of administrative rights is determined by an organization composed of the same people. No matter what the form of the organization is, this principle is the foundation of human social organization, that is, there is no non-human subject at present. With the application of artificial intelligence in administrative governance, whether the intelligent machine provides the decision-making plan for the administrative subject has embodied its own will, whether it violates the traditional administrative ethics of human beings and triggers new security ethics issues. The administrative body should respond to this problem. Pay attention to; when artificial intelligence is used in the process of administrative 
governance, the loss of control of system algorithms may lead to algorithmic discrimination. Algorithmic decisionmaking is inherently predictive, and past discrimination may be solidified in intelligent algorithms and strengthened in the future, resulting in fairness. Ethical issues; the development of artificial intelligence technology complicates the relationship between humans and intelligent machines. How to locate the relationship between humans and intelligent machines is a new ethical issue caused by artificial intelligence, treating artificial intelligence machines as human machines. The tool still recognizes that it has certain consciousness and has "legal personality". At present, the academic circles are still inconclusive, and the administrative subject should consider the relationship between human and intelligent machines in the process of administrative governance.

\section{B. The Regulatory Path Used by Artificial Intelligence in Administrative Governance}

First, the administrative organs should continuously promote the process of artificial intelligence administrative legislation. With the wide application of artificial intelligence in various fields of society and the risks brought by the use of artificial intelligence, administrative law also needs to constantly adjust its research objects and scope to standardize the application of artificial intelligence in administrative governance. At present, China's artificial intelligence law has not yet formed a system. In order to promote the standardization and legalization of artificial intelligence technology in the field of administrative governance, it should be regulated by different levels of legislation such as artificial intelligence. The technical fields of driverless vehicles and intelligent robots have established safety management administrative regulations, laid the foundation for the formulation of relevant laws, further improved relevant laws and regulations, and formulated corresponding rules and judgments according to different industry fields and geographical characteristics. The standards of the case include artificial intelligence into the network security legal supervision system and manage it. In order to prevent administrative inaction and improper supervision, the legalization process of artificial intelligence administrative supervision procedures should be accelerated.

Second, the administrative body should improve the artificial intelligence data security system. Data is the core resource of the era of artificial intelligence, and it is also an important support for the administrative subjects to achieve refined governance and promote the performance of administrative governance. The development of artificial intelligence impacts the traditional administrative governance model. The administrative agencies obtain a large amount of social data information with certain randomness and diversity. The rapid circulation of big data makes the security of data and information not fully guaranteed, and the personal information is extremely easy. In case of leakage and infringement, the administrative organ should optimize the government data process, promote the high matching of government data processes and artificial intelligence technology, attach importance to and optimize the data security system in administrative governance, and back up and archive data information. At the same time, in the face of the objective existence of data barriers, in order to ensure the security of data circulation, the data transmission and preservation methods between administrative agencies should be unified, and the technical obstacles in the data connection between different regions and different administrative departments should be reduced; Establish data management and service centers to achieve openness and sharing of data between administrative agencies and departments, break through the governance bottlenecks such as improper data processing, information mismatch, and inefficient work under the traditional administrative governance model, and improve the efficiency of government operations and government affairs. The quality of the administrative management is "efficient"; on the basis of improving the data security system, the protection of data information is strengthened, and the information about citizens' private data generated in the process of administrative governance is effectively protected and the data is improved.

Then, the administrative governance body should increase the technical research and development of artificial intelligence. At present, the application of artificial intelligence is mainly concentrated in the field of digitalization. The research and development of artificial intelligence technology needs to be strengthened. The application scope of artificial intelligence should be expanded, the research and development of artificial intelligence should be strengthened, and the development of artificial intelligence in administrative governance should be promoted. The application of artificial intelligence technology is based on the operation of big data and algorithms. Large artificial intelligence enterprises with large data resources may bring challenges to social and administrative governance. The development of artificial intelligence needs to strengthen the protection of core data. Data security issues, at the same time, strengthen the dominant position of administrative agencies in the field of artificial intelligence, and prevent corporate entities from intervening in administrative governance by acquiring data. The administrative organ should increase its research and development efforts on the artificial intelligence technology platform, actively cultivate the talents who maintain the artificial intelligence technology platform, and ensure the data and information advantages of the administrative entity to the enterprise. The administrative department can use the existing big data management organization and information management. Based on the department, the department will focus on the internal technical talents and technical forces, and actively develop artificial intelligence technology platforms to achieve independence in artificial intelligence technology. In the development of artificial intelligence technology, it should be noted that the design of artificial intelligence systems should be compatible with the concepts of human dignity and rights, because the generation of intelligent machine systems is always for human beings.

At the same time, the main body of administrative governance needs to improve its governance capacity. The 
application of artificial intelligence in the process of administrative governance has continuously improved the level of administrative governance, but the governance ability of administrative subjects has been weakened to a certain extent. When conducting administrative governance, the administrative body should strengthen the concept of governance and change the organizational model and administration of administrative organs. Run the process to improve your administrative governance. When the administrative governance model is integrated into artificial intelligence technology, it is necessary to realize the profound reform of the administrative organ governance model. It is necessary not only to update the governance concepts of the administrative staff at all levels, but also to establish an open, shared and diversified administrative governance thinking, and to change the existing The administrative organization model and the complex and rigid administrative operation process promote the optimization of the administrative level and realize the flattening of the administrative structure organization. In the administrative governance of the city, regional reconstruction and reorganization between the district administrative agencies in the core areas of the city can be promoted, and the links between administrative agencies can be strengthened to ensure the timeliness and effectiveness of data information transmission. At the same time, it further promotes the intelligentization of administrative operation processes, optimizes the administrative management operation process according to the process problems of artificial intelligence technology application, and improves the efficiency of administrative governance.

Finally, the administrative body needs to pay close attention to the ethical issues caused by artificial intelligence. Although the integration of artificial intelligence into administrative governance has not entered a mature stage, the administrative ethical issues that may arise in the process of its application of technology have begun to emerge. The administrative organization regulates the interactions between people through the algorithm calculation of artificial intelligence technology, but whether the role of artificial intelligence in administrative governance, whether it can represent the authority of administrative subjects, and whether it has the consciousness of self-determination when exercising administrative power, etc. They need to fully discuss them and formulate corresponding rules and regulations. In order to standardize the administrative governance order and solve the administrative ethics problem, the administrative organ must formulate corresponding administrative ethics norms and rules system, carry out the top-level design from the legislative level, construct a complete rule of law system, and clarify the administrative staff's application in the process of artificial intelligence technology, specific responsibilities, correcting the technical dependence behavior in the process of administrative governance, identifying the artificial intelligence technology platform as the technical means of administrative governance, and preventing the administrative entity from shirking responsibility. At the same time, when using intelligent technology in the process of administrative governance, based on the judgment results of the artificial intelligence technology platform, the subjective initiative of the administrative subject should be exerted to realize the unification of the application of intelligent technology and subjective value judgment.

\section{CONCLUSION}

"The emergence of the intelligent revolution has brought an unprecedented crisis and challenge to the current ethical standards, legal rules, social order and public management system." The advent of the artificial intelligence era has made artificial intelligence deeper into the field of administrative governance. The application of artificial intelligence technology has promoted the administrative governance model to become intelligent, and administrative governance has formed a multi-governance pattern in artificial intelligence governance. Artificial intelligence will bring new innovative ideas to administrative governance, and it will bring unprecedented challenges to administrative governance. This paper discusses the current situation and problems of artificial intelligence in the field of administrative governance, aiming to affirm the role of artificial intelligence in administrative governance. And put forward the risk problem of artificial intelligence governance, so as to find the regulatory path of artificial intelligence in administrative governance. The deep integration of artificial intelligence and administrative governance is not only conducive to the rapid development of artificial intelligence, but also contributes to the modernization and refinement of administrative governance. The administrative body should actively adapt to the development trend of artificial intelligence technology and recognize the current administrative governance field. The dilemma is faced with applying the latest scientific and technological achievements to administrative governance, social governance and public services, further updating the governance concept, improving the governance mechanism, innovative governance methods, establishing an intelligent administrative governance system, and modernizing the administrative governance system and governance capacity so as to provide strong technical support.

\section{REFERENCES}

[1] Liu Hongbo and Lin Bin, Research on the mechanism and path of artificial intelligence policy diffusion: a typological perspective. China Administration.

[2] Dong Liren, Research on artificial intelligence development and government governance innovation. Journal of Tianjin Administrative College.

[3] Yuan Xueshi, Constructing a new ecology of "Internet +" administrative law enforcement. Administrative management reform.

[4] Su Lingyin, Seeing "algorithm discrimination" behind artificial intelligence. Chinese Journal of Social Sciences.

[5] Wu Handong, Institutional arrangements and legal regulation in the era of artificial intelligence. Legal science.

[6] Chen Zifu, Alchemy and artificial intelligence: The impact of Hubert Dreyfus on the development of artificial intelligence. Science and Management. 